\title{
Educational opto-mechatronic apparatus to calculate the refractive index of liquids based on Snell's Law
}

U. Rivera Ortega, C. R. Hernandez-Gomez, G. VegaTorres

U. Rivera Ortega, C. R. Hernandez-Gomez, G. Vega-Torres, "Educational opto-mechatronic apparatus to calculate the refractive index of liquids based on Snell's Law," Proc. SPIE 11143, Fifteenth Conference on Education and Training in Optics and Photonics: ETOP 2019, 111432W (2 July 2019); doi: $10.1117 / 12.2519459$ 


\title{
Educational opto-mechatronic apparatus to calculate the refractive index of liquids based on Snell's Law \\ U. Rivera-Ortega ${ }^{\mathrm{a}}$, C. R. Hernandez-Gomez ${ }^{\mathrm{b}}$, G. Vega-Torres ${ }^{\mathrm{b}}$
}

${ }^{a}$ Tecnológico de Monterrey, Escuela de Ingeniería y Ciencias, Ave. Eugenio Garza Sada 2501,

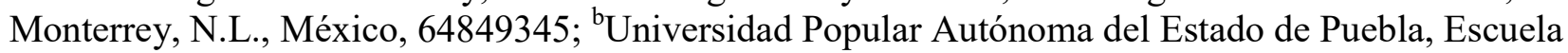
de Ingenierías, Ave. 9 Pte. 1712, Puebla, Pue., México, 72410

\begin{abstract}
This work presents a simple, low-cost opto-mechatronic educational device that determines the refractive index for liquids, which is performed automatically on the computer with an Arduino UNO card through a LabVIEW graphical interface. The operation of the apparatus is based on passing a laser beam through a container with the given substance; then, the refracted beam will be detected with a photoresistor which is moved along the axis by a stepper motor. The distance traveled by the motor until the beam of light impinges on the photoresistor together with known constants (distances between the fixed elements, thickness of the container and refractive index of this material) will be used to calculate the refracted angle through the unknown medium, as well as its refractive index, by linking all the variables in a formula derived from Snell's Law.
\end{abstract}

Keywords: Demonstrative apparatus, refractive index, opto-mechatronic, education

\section{INTRODUCTION}

The refractive index $n$ of a material is a dimensionless number that is related to the speed of light when passing through a dielectric medium ${ }^{1,2}$. This can be seen when light rays traveling from one medium to another bends at the interface. The speed of light in vacuum $(c)$ is approximately $300,000 \mathrm{~km} / \mathrm{s}$, while in another transparent media it is lower than and its represented by $v$, so that $n$ of a material is expressed as

$$
n=\frac{c}{v} .
$$

In a practical approach $n$ can be calculated by using Snell's law ${ }^{3,4}\left(n_{1} \sin \theta_{1}=n_{2} \sin \theta_{2}\right)$, where $n_{1}$ and $n_{2}$ are the refractive indexes of the two different media and $\theta_{1}, \theta_{2}$ are the incidence and refracted angles of a light ray traveling throw them. By following this principle and the use of diffraction gratings ${ }^{5}$, the refractive index of liquids can be calculated by using three diffraction gratings ${ }^{6,7}$, a refractometer based on a sub-micron grating ${ }^{8}$. Some other methods are based on the interference generated by a Michelson interferometer ${ }^{9,10}$.

In the present manuscript a low-cost and automated apparatus designed to estimate the refractive index of liquids is described, which is based on the sensing of the x-displacement of a refracted ray from a laser source when it passed through the testing liquid in accordance with the Snell's law.

\section{METHODOLOGY AND IMPLEMENTATION}

The developed optomechatronic device consists of a generic laser-diode beam that strikes (for implementation simplicity, at an incidence angle of $\theta_{1}=60^{\circ}$ ) the surface of a container with the liquid of the unknown refractive index. The beam of light hits the container and refracts both in the walls of the container and the liquid. Once refracted, a stepper motor moves the photodetector (LDR), which is mounted on a moving platform, until the output beam is detected. The total distance traveled on the x-axis is automatically computed in order to calculate the refractive index (in

Fifteenth Conference on Education and Training in Optics and Photonics: ETOP 2019, edited by

Anne-Sophie Poulin-Girard, Joseph A. Shaw, Proc. of SPIE Vol. 11143, 111432W · C 2019

SPIE, ICO, IEEE, OSA · CCC code: 0277-786X/19/\$18 · doi: 10.1117/12.2519459

Proc. of SPIE Vol. $11143111432 \mathrm{~W}-1$ 
which the entire process as well as the user interface was carried out with LabVIEW and the electronic interface via Arduino UNO microcontroller).

By knowing the distances between the components of the system, as well as the thickness and material of the container, it is possible to calculate the refractive angle of the light in the liquid from the final opening distance. For this calculation, a diagram of the refractions of the light ray in the system is depicted in Figure 1, considering the three media (air, material of the container and given liquid) and all the involved variables to work with Snell's law and trigonometric functions.

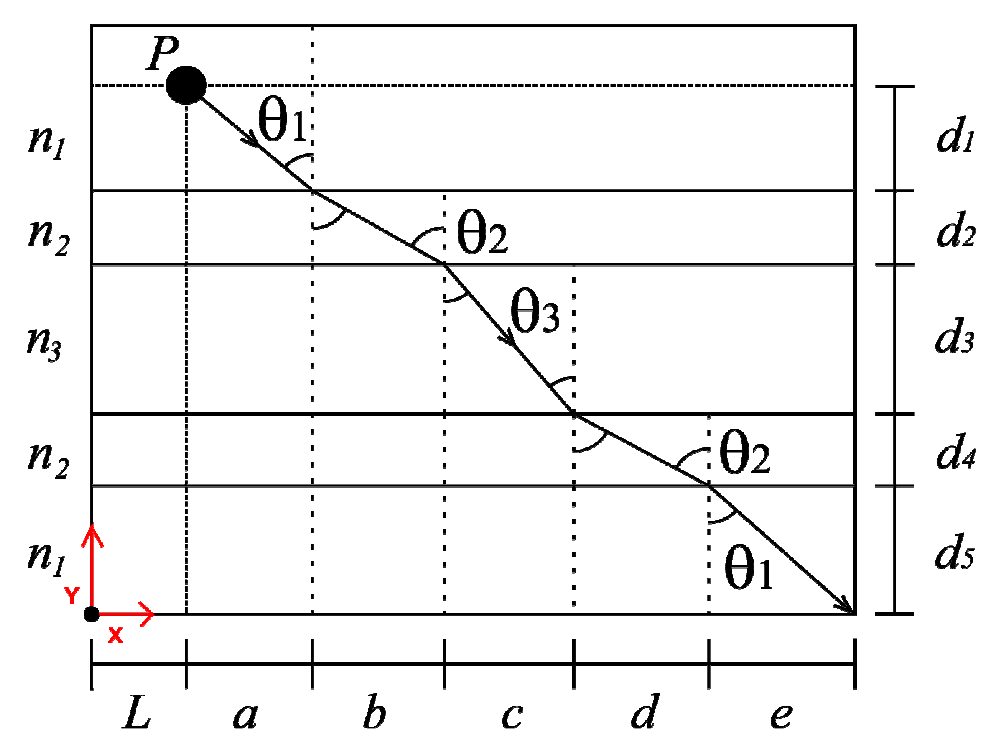

Figure 1. Diagram of the incidence and refraction angles of the laser's beam through different materials.

According to the ray diagram and the user defined assembly, the known constants are the angle of incidence $\theta_{1}$ (starting from point $\mathrm{P}$ ), the refractive indices of the air $n_{1}$ and the material of the container $n_{2}$ (which is considered as 1.49 since the material is acrylic); the vertical segments: the distance between the origin of the light beam and the container $d_{1}$, the thicknesses of the walls of the container $d_{2}$ and $d_{4}$, the opening of the container $d_{3}$ and the distance between the container and the receiver of the refracted light $d_{5}$; as well as the initial distance $(L)$ traveled by the motor on the x-axis until it perpendicularly aligns to point $P$.

The cathetus $(a, \ldots, \mathrm{e})$ opposite to each angle (or the horizontal distances of each light segment) are defined now:

$$
\begin{aligned}
& \frac{a}{d_{1}}=\tan \theta_{1} \therefore a=d_{1} \tan \theta_{1} \\
& \frac{b}{d_{2}}=\tan \theta_{2} \therefore b=d_{2} \tan \theta_{2}, \\
& \frac{c}{d_{3}}=\tan \theta_{3} \therefore c=d_{3} \tan \theta_{3},
\end{aligned}
$$




$$
\begin{aligned}
& \frac{d}{d_{4}}=\tan \theta_{2} \therefore d=d_{4} \tan \theta_{2}, \\
& \frac{e}{d_{5}}=\tan \theta_{1} \therefore e=d_{5} \tan \theta_{1} .
\end{aligned}
$$

Snell's law was also introduced for the relevant diffraction changes of the medium: from the air to the material of the container and from this to the contained liquid

$$
\begin{aligned}
& n_{1} \operatorname{sen} \theta_{1}=n_{2} \operatorname{sen} \theta_{2}, \\
& n_{2} \operatorname{sen} \theta_{2}=n_{3} \operatorname{sen} \theta_{3},
\end{aligned}
$$

Clearing $\theta_{2}$ from Eq. (7)

$$
\theta_{2}=\arcsin \left(\frac{n_{1} \operatorname{sen} \theta_{1}}{n_{2}}\right)
$$

and replacing in Eq. (3) and Eq. (5) to obtain

$$
\begin{aligned}
& b=d_{2} \tan \left[\arcsin \left(\frac{n_{1} \operatorname{sen} \theta_{1}}{n_{2}}\right)\right], \\
& d=d_{4} \tan \left[\arcsin \left(\frac{n_{1} \operatorname{sen} \theta_{1}}{n_{2}}\right)\right] .
\end{aligned}
$$

The total distance in the $\mathrm{x}$-axis that the light beam travels is what the system will measure and is equal to the sum of the horizontal distances

$$
D=L+a+b+c+d+e .
$$

Replacing Eq. (12) with Eqs. (2), (10), (4), (11) and (6) for $a, b, c, d, e$, we get

$$
D=L+d_{1} \tan \theta_{1}+d_{2} \tan \left[\arcsin \left(\frac{n_{1} \operatorname{sen} \theta_{1}}{n_{2}}\right)\right]+d_{3} \tan \theta_{3}+d_{4} \tan \left[\arcsin \left(\frac{n_{1} \operatorname{sen} \theta_{1}}{n_{2}}\right)\right]+d_{5} \tan \theta_{1} .
$$

Clearing $\theta_{3}$ from Eq. (13)

$$
\theta_{3}=\operatorname{atan}\left(\frac{1}{d_{3}}\left\{D-L-\left(d_{1}+d_{5}\right) \tan \theta_{1}-\left(d_{2}+d_{4}\right) \tan \left[\arcsin \left(\frac{n_{1} \operatorname{sen} \theta_{1}}{n_{2}}\right)\right]\right\}\right)
$$


and clearing $n_{3}$ from Eq. (8)

$$
n_{3}=\frac{n_{2} \operatorname{sen} \theta_{2}}{\operatorname{sen} \theta_{3}}
$$

Replacing Eq. (6) in Eq. (14)

$$
n_{3}=\frac{n_{1} \operatorname{sen} \theta_{1}}{\operatorname{sen} \theta_{3}}
$$

From Eq. (16), the designed LabVIEW to obtain the refractive index after evaluating Eq. (14) to get the resulting angle. The schematic setup is shown in Figure 2, in which the most important components such as: a generic laser source $(620 \mathrm{~nm})$, the container, the stepper motor and the light sensor mounted over a translation stage are indicated (the complete procedure can be observed in Media1).
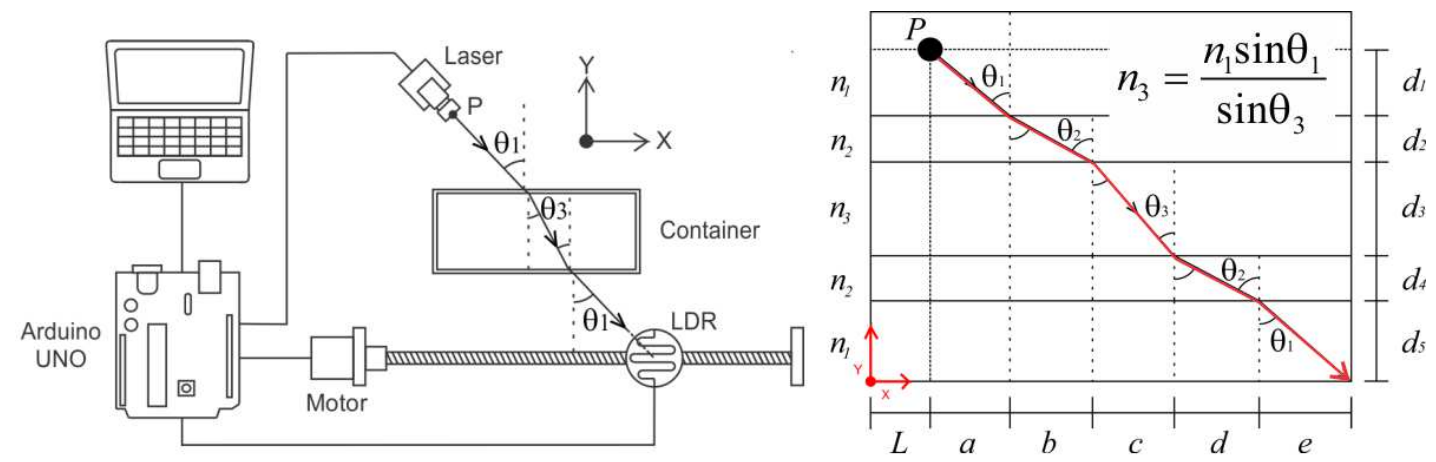

$$
\theta_{3}=\operatorname{atan}\left(\frac{1}{d_{3}}\left\{D-L-\left(d_{1}+d_{5}\right) \tan \theta_{1}-\left(d_{2}+d_{4}\right) \tan \left[\arcsin \left(\frac{n_{1} \sin \theta_{1}}{n_{2}}\right)\right]\right\}\right)
$$

Figure 2. Schematic diagram of the apparatus, the ray tracing and formula to calculate $\theta_{3}$.

In order to validate the performance of the optomechatronic device, several tests (35 per sample) were carried out to calculate the refractive index of distilled water, ethanol and glycerin. The obtained data was compared with the experimental value given by a refractometer (ATAGO Pal-1), except for glycerin, whose refractive index could not be measured with this apparatus. Nevertheless, for the latter, a theoretical value of $n=1,473$ was considered. These results are presented in Figures 3-4.

For the distilled water a refractive index of $n=1,3317$ was measured with the commercial refractometer and the measurements made with the proposed apparatus showed an average of $n=1,3347$, that is an error of $0,23 \%$. On the other hand, ethanol presented a refractive index of $n=1,3609$ and the average measurement was $n=1,3616$, whose error of $0,05 \%$ is even lower than the one of the fatter. In addition, it is important to mention that the measurements of both tests showed a standard deviation of $\sigma= \pm 0,012$. Regarding to glycerin, the measurements made gave an average of $n=1,4675$, with a standard deviation of $\sigma= \pm 0,02$. In this way, an error of $0.37 \%$ was obtained 

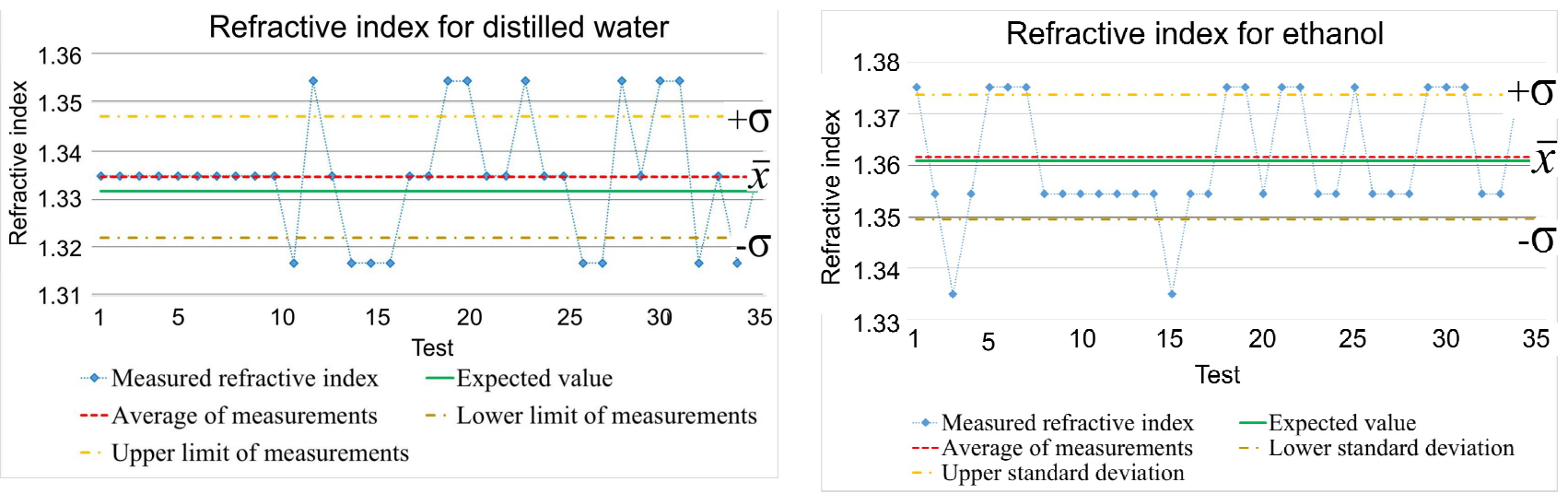

Figure 3. Measured refractive index for distilled water and ethanol.

For the distilled water a refractive index of $n=1,3317$ was measured with the commercial refractometer and the measurements made with the proposed apparatus showed an average of $n=1,3347$, that is an error of $0,23 \%$. On the other hand, ethanol presented a refractive index of $n=1,3609$ and the average measurement was $n=1,3616$, whose error of $0,05 \%$ is even lower than the one of the fatter. In addition, it is important to mention that the measurements of both tests showed a standard deviation of $\sigma= \pm 0,012$. Regarding to glycerin, the measurements made gave an average of $n=1,4675$, with a standard deviation of $\sigma= \pm 0,02$. In this way, an error of $0.37 \%$ was obtained.

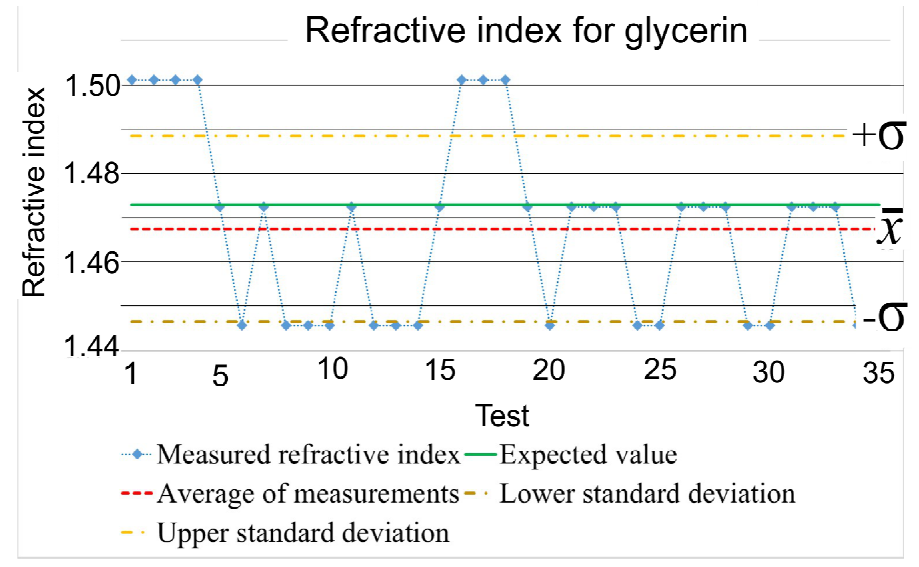

Figure 4. Measured refractive index for glycerin.

\section{CONCLUSIONS}

In this manuscript it has been presented a simple low-cost educational optomechatronic apparatus that computes the refractive index of liquids inside of an acrylic container, which is based on the application of the Snell's law by measuring the $\mathrm{x}$-distance in which the output beam is refracted. The sensing of the beam was achieved by a photoresistor (LDR) attached to a platform and moved by a stepper motor, controlled by Arduino UNO using a LabVIEW interface. 
The refraction indexes of three liquids have been measured: ethanol, water and glycerin. The validation of the procedure was carried out by comparing the results with the ones measured by a commercial refractometer and depicted in Figs. (34).

It is worth mentioning that the value of this device is lower than $\$ 54.99$ USD. In spite of this, the average measurement of the refractive index of any given substance is not further than $0,22 \%$ of the real value. Moreover, the accuracy of the proposed setup depends on the number of steps given by the stepper motor, as well as on the distance between threads in the translation screw which moves the LDR sensor.

\section{REFERENCES}

[1] Hecht, E. [Optics], 3rd ed., Addisson Wesley, San Francisco, CA, (1998).

[2] Malacara, D. [Optica básica], 1st ed., S.L. Fondo de Cultura Económica de España, México, DF, (2006).

[3] Pedrotti F. L. and Pedrotti L. S., [Introduction to Optics] , 2nd ed., Prentice Hall, New Jersey, (1993).

[4] Fowless G. R., [Introduction to Modern Optics Introduction to Optics] , 2nd ed., Dover Publications, Inc, New York, (1975).

[5] Larouche, S. and Smith D. R., "Reconciliation of generalized refraction with diffraction theory," Opt. Lett. 12, 2391-2393 (2012).

[6] Singh, S., "Three Gratings System for the Measurement of Refractive Index of Liquids," Int. J. Appl. Sci. Math. 3, 115-116 (2016).

[7] Durán-Ramírez, V. M., Martínez-Ríos, A., Guerrero-Viramontes, J. A., Muñoz-Maciel, J., Peña-Lecona, F. G., Selvas-Aguilar, R., Anzueto-Sánchez, G., "Measurement of the refractive index by using a rectangular cell with a fs-laser engraved diffraction grating inner wall," Opt. Express. 24, 29899-29906 (2014).

[8] Chun-Wei, L., Chun-Che, W., Shih-Chieh, L., "A simple and wide-range refractive index measuring approach by using a sub-micron grating", Appl. Phys. 106, 151907-1 151907-4 (2015).

[9] Kachiraju, S. R., Gregory, D. A., "Determining the refractive index of liquids using a modified Michelson interferometer," Opt. Laser Technol. 44, 2361-2365 (2012).

[10] Dobbins, H. M., Peck E. R., "Change of refractive index of water a function of temperature," J. Opt. Soc. Am. 63, 318-320 (1973). 